schizophrenia and bipolar disorder as two separate conditions. This separation is respected by drug companies, regulators, research funders, journals and bench researchers. Add that lot up, and you get a fundamental problem with psychiatry.

Next month, the American Psychiatric Association will release the long-awaited fifth version of its Diagnostic and Statistical Manual of Mental Disorders (DSM-5), which lists mental illnesses and their symptoms. Work on preparing the DSM-5 has been clouded in controversy, and the arguments over which conditions should have been included and which left out will rumble on for some time.

The more fundamental problem, as the News Feature explores, is growing doubt about the way the DSM-5 classifies mental disorders. Psychiatrists have long known that the illnesses of patients they see in the clinic cannot be broken down into discrete groups in the way that is taught at medical school. Symptoms overlap and flow across diagnostic boundaries. Patients can show the signs of two or three disorders at the same time. Treatments are inconsistent. Outcomes are unpredictable.

Science was supposed to come to the rescue. Genetics and neuroimaging studies would, all involved hoped, reveal biological signatures unique to each disorder, which could be used to provide consistent

and reliable diagnoses. Instead, it seems the opposite is true. The more scientists look for biomarkers for specific mental disorders, the harder the task becomes. Scans of the DNA and brain function of patients show the same stubborn refusal to group by disease type. Genetic risk factors and dysfunction in brain regions are shared across disorders.

"Patients'

illnesses cannot

be broken down

into discrete

groups in

the way that

is taught at medical school."
Psychiatrists joke that their patients have not read the textbooks. The reality is serious and more troubling - the textbook is wrong.

The American Psychiatric Association routinely points out that its DSM disease categories are intended only as diagnostic tools. It does not claim that they mark genuine biological boundaries. But the system is set up as if they do. That might explain why biomarkers and new drugs for mental illness remain elusive. The system should change. Funders and journals must encourage work that cuts across the boundaries. Researchers should be encouraged to investigate the causes of mental illness from the bottom up, as the US National Institute of Mental Health is doing. The brain is complicated enough. Why investigate its problems with one hand tied behind our backs?
ANNOUNCEMENT

\section{Reducing our irreproducibility}

$\mathrm{O}$ ver the past year, Nature has published a string of articles that highlight failures in the reliability and reproducibility of published research (collected and freely available at go.nature.com/ huhbyr). The problems arise in laboratories, but journals such as this one compound them when they fail to exert sufficient scrutiny over the results that they publish, and when they do not publish enough information for other researchers to assess results properly.

From next month, Nature and the Nature research journals will introduce editorial measures to address the problem by improving the consistency and quality of reporting in life-sciences articles. To ease the interpretation and improve the reliability of published results we will more systematically ensure that key methodological details are reported, and we will give more space to methods sections. We will examine statistics more closely and encourage authors to be transparent, for example by including their raw data.

Central to this initiative is a checklist intended to prompt authors to disclose technical and statistical information in their submissions, and to encourage referees to consider aspects important for research reproducibility (go.nature.com/oloeip). It was developed after discussions with researchers on the problems that lead to irreproducibility, including workshops organized last year by US National Institutes of Health (NIH) institutes. It also draws on published concerns about reporting standards (or the lack of them) and the collective experience of editors at Nature journals.

The checklist is not exhaustive. It focuses on a few experimental and analytical design elements that are crucial for the interpretation of research results but are often reported incompletely. For example, authors will need to describe methodological parameters that can introduce bias or influence robustness, and provide precise characterization of key reagents that may be subject to biological variability, such as cell lines and antibodies. The checklist also consolidates existing policies about data deposition and presentation.

We will also demand more precise descriptions of statistics, and we will commission statisticians as consultants on certain papers, at the editor's discretion and at the referees' suggestion.

We recognize that there is no single way to conduct an experimental study. Exploratory investigations cannot be done with the same level of statistical rigour as hypothesis-testing studies. Few academic laboratories have the means to perform the level of validation required, for example, to translate a finding from the laboratory to the clinic. However, that should not stand in the way of a full report of how a study was designed, conducted and analysed that will allow reviewers and readers to adequately interpret and build on the results.

To allow authors to describe their experimental design and methods in as much detail as necessary, the participating journals, including Nature, will abolish space restrictions on the methods section.

To further increase transparency, we will encourage authors to provide tables of the data behind graphs and figures. This builds on our established data-deposition policy for specific experiments and large data sets. The source data will be made available directly from the figure legend, for easy access. We continue to encourage authors to share detailed methods and reagent descriptions by depositing protocols in Protocol Exchange (www.nature.com/ protocolexchange), an open resource linked from the primary paper.

Renewed attention to reporting and transparency is a small step. Much bigger underlying issues contribute to the problem, and are beyond the reach of journals alone. Too few biologists receive adequate training in statistics and other quantitative aspects of their subject. Mentoring of young scientists on matters of rigour and transparency is inconsistent at best. In academia, the ever increasing pressures to publish and chase funds provide little incentive to pursue studies and publish results that contradict or confirm previous papers. Those who document the validity or irreproducibility of a published piece of work seldom get a welcome from journals and funders, even as money and effort are wasted on false assumptions.

Tackling these issues is a long-term endeavour that will require the commitment of funders, institutions, researchers and publishers. It is encouraging that NIH institutes have led community discussions on this topic and are considering their own recommendations. We urge others to take note of these and of our initiatives, and do whatever they can to improve research reproducibility. 